\title{
Visual Analysis of Vehicle Trajectories for Determining Cross-Sectional Load Density
}

\author{
Roman Juránek ${ }^{a}$, Jakub Špaňhel ${ }^{a}, J_{a k u b}$ Sochor ${ }^{a}$, Adam Herout $^{a}$, Jan Novák ${ }^{b}$ \\ ${ }^{a}$ GRAPH@FIT, Brno University of Technology, Božetěchova 1/2, Brno 612 66, Czech Republic \\ ${ }^{b}$ CDV-Transport Research Centre, Lišeňská 33a, Brno 636 00, Czech Republic
}

\begin{abstract}
The goal of this work was to analyze the behavior of drivers on third class roads with and without horizontal lane marking. The roads have low traffic volume, and therefore a conventional short-term study would not be able to provide enough data. We used recording devices for long-term (weeks) recording of the traffic and designed a system for analyzing the trajectories of the vehicles by means of computer vision. We collected a dataset at 6 distinct locations, containing 1010 hours of day-time video. In this dataset, we tracked over 12000 cars and analyzed their trajectories. The results show that the selected approach is functional and provides information that would be difficult to mine otherwise. After application of the horizontal markings, the drivers slowed down and shifted slightly towards the outer side of the curve.
\end{abstract}

KEYWORDS: Road Safety, Lane Marking, Trajectory Analysis, Computer Vision, Vehicle Tracking

\section{INTRODUCTION}

The principal assumption of this work is that the driver controls the vehicle according to previous stimuli coming from the roadway. Based on these, the driver decides to adjust the path and/or speed of the vehicle. It is known that the driver approaching a narrow part of the roadway decelerates (Jamieson, 2012). The speed together with the reaction times of the driver further influence the trajectory of the vehicle, having a direct impact on conflict situations and traffic accidents.

Dangerous situations often occur in horizontal curves with smaller radii, where the roadway is narrowed, and the drivers are acting recklessly (do not decrease speed). Widening the road or completely changing its location would be expensive and often unfeasible solution to such a problem. A low-cost solution that could help to some extent would be horizontal traffic marking on the road, either in the form of a shoulderline or as the centerline between the lanes. The question is which of these (or their combination) is more suitable/influential and what effect can be expected from their application.

In this work, we used long-term visual recording and computer vision analysis of the recorded videos to answer these questions. It turns out that computer vision and visual analysis of the traffic can provide answers to questions related to transportation safety and improve the situation with very low costs. The gained information could otherwise be very hard to obtain, especially when the locations of interest were situated on third class roads with very low traffic volume, where human observation would be inefficient or impossible. Also, the cost-effectiveness of the proposed solution was even more dominant on these roads.

Our measurements were related to the density of traffic across the lateral cross-section of the road in the horizontal curve as dependent on the horizontal lane markings. It is the distance of the vehicle from the road side along with its speed which can provide evidence of the influence that the presence of the central/shoulder lane markings might have on the trajectory of the vehicle. The assumption is that the drivers keep a distance from these markings and try to adjust their driving trajectory.

On several analyzed locations we collected long video recordings before and after horizontal road markings were applied, and processed the videos. First, we calibrated the cameras to enable measurements on the road plane. We detected and tracked passing vehicles and measured their position in three road cross sections. Finally, we analyzed how the distribution of car speeds and positions changes with 


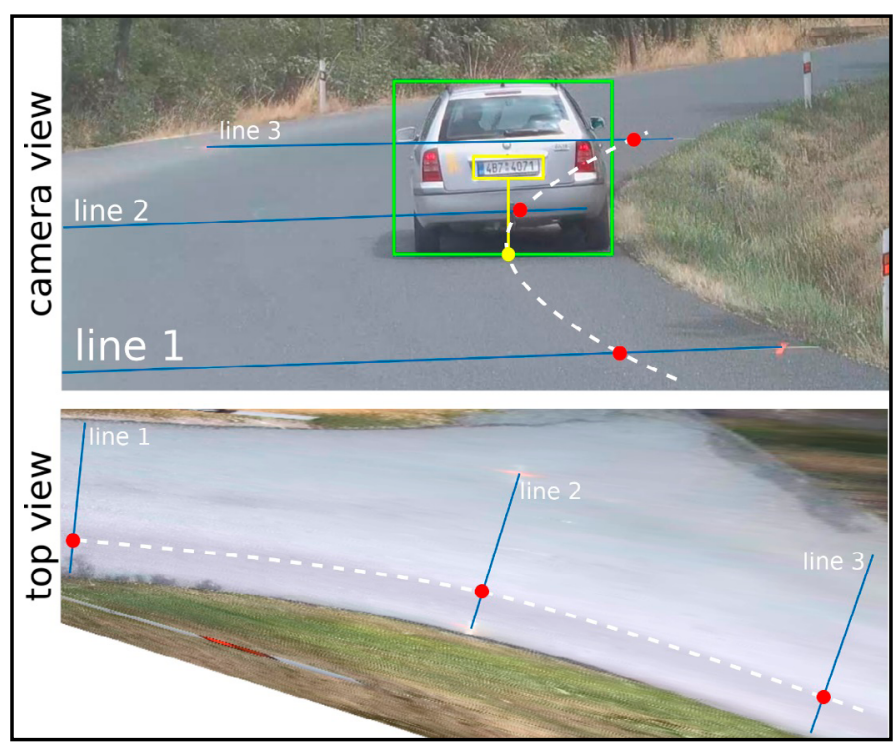

Fig. 1: We detect and track cars in videos and record the lateral position of cars on three road cross sections. We analyze how driver behavior changes when horizontal line markings are drawn.

the presence of the road markings. The results show that the proposed approach indeed can mine interesting data on roads with very low traffic volume, where manual processing would not be suitable. The change of behavior of the drivers after the horizontal line marking was painted was not tremendous. Still, after application of the horizontal marking, the drivers visibly slowed down, their trajectories moved slightly towards the outer side of the curve. In the cases when the central line was painted (contrary to those where shoulder-lines were drawn), the vehicles tended to drive closer towards the central curve of their traffic lane.

\section{BACKGROUND}

Among the first ones to be interested in the position of the vehicle in the lateral cross-section of the road were Glennon and Weaver, (2013) (cited by Fitzsimmons et al., (2013)). They made a video recording and an analysis of the vehicles' behavior at five curved road sections. Their study identified that the vehicles tend to travel through the curve along a straight line and later suddenly change their direction.

Jamieson, (2012) carried out a study on seven curves with a diameter smaller than $300 \mathrm{~m}$. They analyzed at least 100 trajectories of vehicles by observing video recordings. Jamieson sorted the drivers' behavior into four categories:
- drivers who keep in the center of their driving lane (Mid-Lane),

- drivers who enter the curve by the left side of their lane and leave by the right side (Left In Right Out),

- drivers who enter the curve by the right side of their lane and leave by the left side (Right In Left Out),

- drivers who reduce the curvature by driving on straighter trajectory (Cutting).

Jamieson, (2012), based on multiple studies, made further conclusions:

- drivers tend to straighten their trajectory when passing through a curve,

- the trajectory of the vehicle usually moves towards the center of the curvature (depending on the radius),

- drivers' behavior is based on conscious and unconscious decision making,

- one trajectory (passage of a vehicle through the given curve) is unique, depending on the personality of the driver,

- speed and consequent centrifugal force also depends on the driver,

- loss of control of the vehicle in the curve cannot be judged only based on the speed, but the lateral position must also be considered.

Chrysler et al., (2011) verified that drivers do not move on the standard geometric curves corresponding to the shape of the road, but rather on flattened curves. They cited the study by Zador et al., (1987) who found out that in left curves, the drivers found themselves closer to the central line, and in right curves, they drove closer to the shoulder line. In their study, Chrysler et al. used a piezoelectric sensor placed on the road in the " $Z$ " shape.

\subsection{Models of Accident Rates Based on the Vehicle's Lateral Position}

The study by Chrysler et al. (2011) identified a model based on observing 32 curves. They found out that the number of traffic accident increased with the distance of the vehicle's trajectory from the center of the driving lane and with the change of the lateral vehicle's position. Their study confirmed the models by Pagano (1972) and by Stimson et al. (2009). 


\subsection{Related Work in Visual Processing of Traffic}

In order to analyze the video recordings, we employ computer vision techniques - object detection and tracking. Object detection methods localize object instances in the image. One of the most widespread methods is Boosted Soft-cascade (Dollár et al., 2014) targeted to detection of pedestrians and originating from an older Classifier Cascade (Viola et al., 2004). This method can detect rigid objects (such as license plates in this paper). Recently, many methods based on convolutional neural networks appeared (Liu et al., 2016; Redmon et al., 2017; Ren at al., 2015). These are suitable for detection of complex, non-rigid objects and they can also reliably detect bounding boxes of the objects. Tracking methods are based either on optical flow (Shi et al., 1994) or they search for movement of object instances in subsequent frames by learning object appearance (Babenko et al., 2011; Henriques et al., 2015). Another class of tracking methods is Tracking by Detection (Murray, 2017) which relies on the underlying object detector and associates the detections in time.

\section{METHODOLOGY}

We installed a static camera at each location and visually captured several days of traffic before and after road markings were drawn. It should be noted that our purpose was to analyze lower-class roads with low traffic volume, and the period of multiple days was required in order to capture a sufficient number of trajectories. This section describes how video data are processed and how trajectories were analyzed. In our experiments presented in Section 4, we describe the analyzed locations and discuss the results.

\subsection{On-site preparations}

In order to measure distances in the captured image space, the camera needs to be calibrated (including the scale). After camera installation, the operators marked several clearly visible points on the road surface and measured their distances (see Figure 3 for an example of such a scene). We define three virtual cross-sections in which the positions of the passing cars are being measured and evaluated. Figure 3 shows a camera view from one of the analyzed locations with virtual lines and measured distances. After camera in- stallation, the speed of several cars was measured by a hand-held radar in order to get reference speeds for validating the visual vehicle tracker.

\subsection{Camera calibration}

From the marker locations $\boldsymbol{p}_{i}$ in camera image (annotated manually) and their distances $d_{i j}$, we recovered locations of markers on the road plane $\boldsymbol{P}_{\boldsymbol{i}}$ (see Figure 2). To estimate the homogeneous positions of the markers in the road plane $\boldsymbol{P}_{i}$, we first set $\boldsymbol{P}_{\mathbf{1}}=(0,0,1)^{T}$ and $\boldsymbol{P}_{2}=\left(0, d_{12}, 1\right)^{T}$. Then, it is possible to construct other points $\boldsymbol{P}_{\boldsymbol{i}}$ always as an intersection of two circles. Formally, point $\boldsymbol{P}_{3}$ is computed as:

$$
\boldsymbol{P}_{3}=\arg \min _{\boldsymbol{P}} \sum_{j \in\{1,2\}}\left|d_{j 3}-\left\|P_{j}-\mathrm{P}\right\|\right|
$$

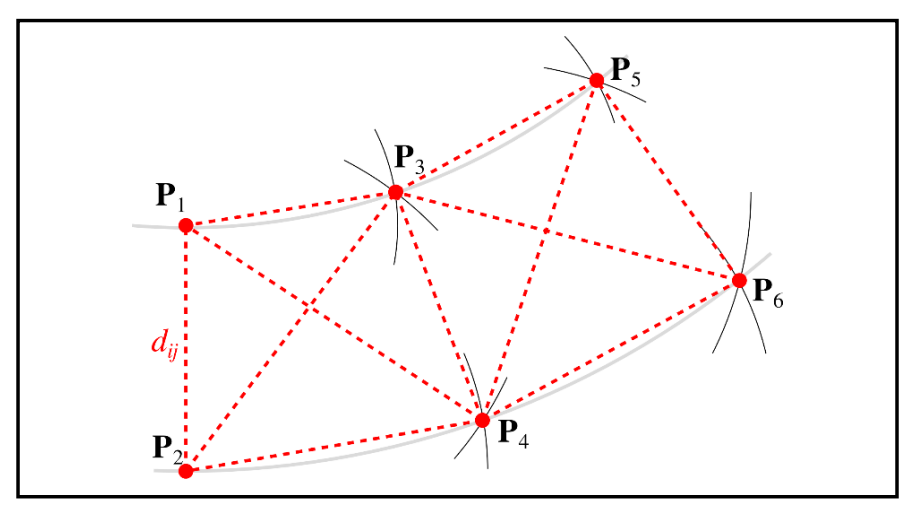

Fig. 2: Construction of road plane coordinates $P_{i}$ based on the measured distances $d_{i j^{\circ}}$

As the two circles have two intersections, the ones with higher $x$ coordinate are used. Following this procedure, the rest of the points are constructed. Finally, as distances $d_{34}$ and $d_{56}$ are not used for the construction of the points, we used them as validation measurements and the relative error was approximately $1 \%$.

To construct the mapping between the image space and the road plane, 2D homography $\boldsymbol{H}$ is computed between annotated positions in the image space $\boldsymbol{p}_{i}$ and the corresponding points on the road plane $\boldsymbol{P}_{i}$. Since there are 6-point correspondences, the linear system is over-determined and linear least squares are used to estimate the homography.

In this step, the scene is assumed to be approximately planar - this condition must be locally satisfied at least in the measured area. After this calibration, any image point can be mapped to its position on the road plane, and the real-world distances between image points can thus be measured. 


\subsection{Video processing}

The captured video was extremely long - tens of hours of Full HD recording. We divided it into one-hour segments and discarded night-time videos (as it was impossible to track cars in such videos reliably). In each video, vehicles' license plates were detected using custom trained ACF detector (Dollár et al., 2014), and the vehicles themselves by using Faster R-CNN (Ren et al., 2015). The reason behind using two different detectors is that ACF is very fast and it can reliably detect the presence of a vehicle by detecting its license plate. Then, on frames with a car present, the accurate car bounding box is detected by Faster $\mathrm{R}-\mathrm{CNN}$ which, despite its name, is very slow even on recent GPUs (approx. 6 fps on Full HD video). Using ACF for detection of the car bounding box is not suitable since it cannot reliably detect objects of such variability (cars from arbitrary viewpoint) and estimate their bounding box. On the other hand, CNN-based detectors (Liu et al. 2016; Redmon et al., 2017; Ren et al., 2015) are known to solve the task well.

Car detections were tracked by a Kalman filter, and license plate detections were associated with tracks. To measure the position of a car in the road plane, we simply project the center of its license plate to the bottom edge of the car's bounding box and transform this point using homography $\boldsymbol{H}$ (defined in Sec. 3.2). Formally, car track $T$ of length $N_{T}$ is a sequence of image points $x_{i}$ transformed with the calibration homography $\boldsymbol{H}$ to the road plane, associated with a timestamp $t$.

$$
T=\left\{\left(\boldsymbol{H} \boldsymbol{x}_{i}, t_{i}\right) \mid i=1 \ldots N_{T}\right\}
$$

To remove noise from the tracking, positions $H x_{i}$ are filtered by a third order polynomial and the interpolated track $\mathrm{S}$ is obtained.
Table 1: Recording length (hours) and the number of car observations per location

\begin{tabular}{lcc}
\hline & Length $[\mathrm{h}]$ & \# Observations \\
\hline Location 1 & 194 & 1414 \\
Location 2 & 179 & 2844 \\
Location 3 & 220 & 1759 \\
Location 4 & 228 & 695 \\
Location 5 & 114 & 1166 \\
Location 6 & 75 & 4824 \\
Total & 1010 & 12702 \\
\hline
\end{tabular}

For a trajectory $S$, crossing points $l_{i}$ with the road cross sections $j \in\{1,2,3\}$ are obtained. By measuring the traveled distance on the road plane and the time difference, average speed $v$ between the first and the last cross section is determined. Finally, for purposes of statistics, a trajectory is represented by its speed and the three positions on the cross sections $T_{k}=\left(l_{j}, v\right), j \in\{1,2,3\}$.

\section{RESULTS}

We analyzed 6 locations on third class roads. On each location, a static camera was installed several days before line markings were drawn and recorded the traffic before and after line marking application. Table 1 summarizes the video lengths and traffic statistics for each location. In total, the study includes 1010 hours of traffic video recording.

Although the cameras were meant to be static, long recording showed the slight drift of the camera image due to wind and instability of camera mounting. Therefore, we used different sets of annotated

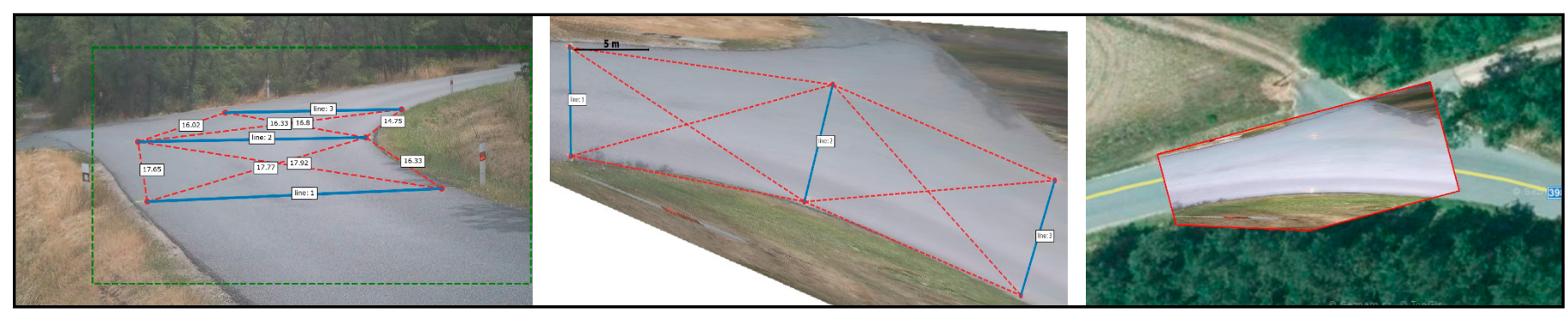

Fig. 3: An example of the calibration markings and measurements. A minimal of 6 points, three on each side of the road are marked on the side of the road, and the mutual distances are measured in the real world. Left: camera image where positions of markers were defined, middle: birds-eye view of the scene rendered using homography recovered form point distances, right: our reconstruction of the road overlayed on orthographic map shows that the reconstruction is correct. 
marker locations for video before and after the road marking application. On Location 2, the recording was affected by the severe wind which caused camera vibrations and subsequent camera image shaking. This image shaking resulted in a significant error in the measurements of car positions (where the assumption was that camera image is fixed). We did not find any reliable method of how to automatically stabilize the image to make the position of markers fixed. We include the results from this location; however, they are not to be considered significant.

The individual scenes included in the study are visualized in Figure 4. The illustration shows an illustrative frame from the video before and after the application of the horizontal line markings. It also shows the camera view re-projected to the horizontal plane based on the calibration points. In these views, the three cross-section lines are shown as well as a visualization of the distribution of cars going in each direction before (blue) and after (orange) the application of the horizontal line markings.

More detailed histograms of the vehicles' lateral positions within section 2 (the middle one) and the vehicles' speeds are shown in Figure 5. The lateral shifts in the vehicles' position are more significant than the change in speed of the vehicles. Table II shows detailed information about the locations, the number of observed vehicles and the average speed individually for the inner and outer driving lanes. Finally, Figure 6 visualizes the change in speed and lateral position within the cross-sections together for all the locations (separately reporting the inner and the outer driving lane).

We tested the statistical significance of the difference of the measurements by Welch's t-test and Kolmogorov-

Smirnov test. The two-sided test, with the null hypothesis, that two independent samples were drawn from the same continuous distribution indicated that 5 of 12 -speed measurements and 7 of 12 lateral positions significantly differed (KolmogorovSmirnov test, $p$-value 5\%). The two-sided test, with the null hypothesis, that two independent samples have identical mean value reported that 7 of 12 -speed distributions and 10 of 12 were significantly different (Welch's test, $p$-value 5\%).

\section{CONCLUSIONS}

We presented research on the impact of horizontal line markings on the behavior of drivers in curves on third class roads. The traffic volume is so low in this road category that a conventional way of measuring the data (an observer at the spot, manual analysis of video recordings, etc.) would fail. Usage of long-term video recording and an efficient visual analyzer allows obtaining empirical data about the behavior of the drivers that may improve driving safety by taking appropriate and cost-efficient measures.

Table 2: Details of the Measurements at Different Locations. Average speed is in $\mathrm{km} / \mathrm{h}$.

\begin{tabular}{|c|c|c|c|c|}
\hline & Markings & Curve & \# Vehicles & Avg. Speed \\
\hline \multirow{4}{*}{ نَత } & $x$ & Inner & 131 & 66.1 \\
\hline & $\checkmark$ & Inner & 41 & 71.7 \\
\hline & $x$ & Outer & 844 & 66.3 \\
\hline & $\checkmark$ & Outer & 367 & 69.1 \\
\hline \multirow{4}{*}{ 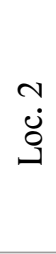 } & $\times$ & Inner & 777 & 70.3 \\
\hline & $\checkmark$ & Inner & 709 & 63.7 \\
\hline & $x$ & Outer & 673 & 66.7 \\
\hline & $\checkmark$ & Outer & 621 & 64.9 \\
\hline \multirow{4}{*}{ 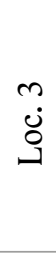 } & $x$ & Inner & 255 & 61.3 \\
\hline & $\checkmark$ & Inner & 307 & 61.6 \\
\hline & $\times$ & Outer & 520 & 61.5 \\
\hline & $\checkmark$ & Outer & 667 & 60.0 \\
\hline \multirow{4}{*}{$\begin{array}{l}+ \\
\dot{\jmath} \\
\dot{\jmath}\end{array}$} & $\times$ & Inner & 352 & 48.5 \\
\hline & $\checkmark$ & Inner & 222 & 47.9 \\
\hline & $x$ & Outer & 51 & 48.6 \\
\hline & $\checkmark$ & Outer & 61 & 47.4 \\
\hline \multirow{4}{*}{ n } & $x$ & Inner & 308 & 56.9 \\
\hline & $\checkmark$ & Inner & 201 & 55.0 \\
\hline & $x$ & Outer & 382 & 56.7 \\
\hline & $\checkmark$ & Outer & 274 & 55.1 \\
\hline \multirow{4}{*}{$\begin{array}{l}0 \\
ن \\
0 \\
0\end{array}$} & $x$ & Inner & 1201 & 62.5 \\
\hline & $\checkmark$ & Inner & 979 & 64.1 \\
\hline & $x$ & Outer & 1690 & 61.7 \\
\hline & $\checkmark$ & Outer & 885 & 62.0 \\
\hline
\end{tabular}

The results show that after the lines were drawn, the drivers tended to slow down and to move slightly (around $20 \mathrm{~cm}$ ) towards the outer side of the curvature. When the center line was drawn (contrary to the situation when the side line markings were drawn), the cars were less spread across the driving lane, i.e., 
the drivers generally stayed closer to the ideal center line of the driving lane.

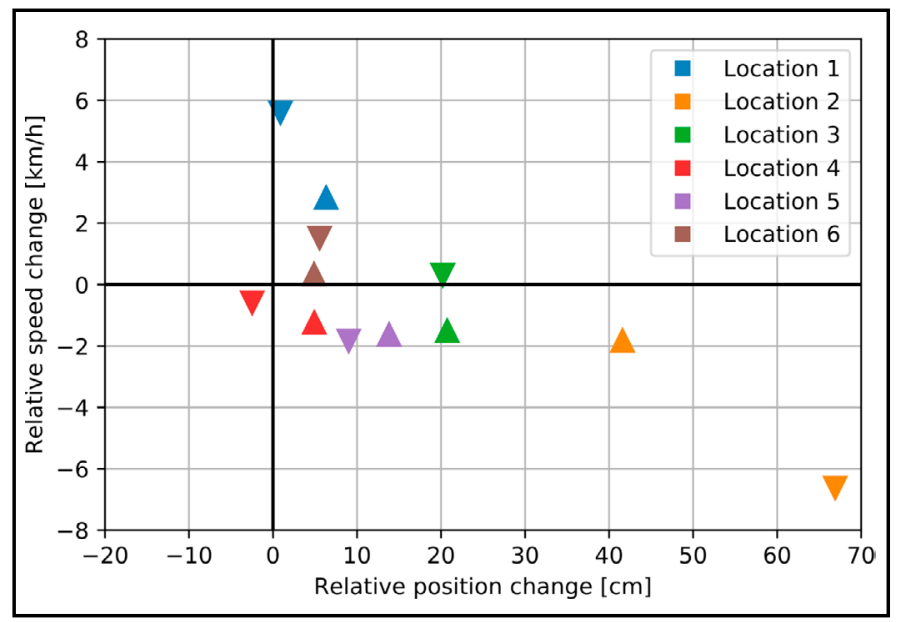

Fig. 6: Change of mean car position (horizontal axis) and mean car speed (vertical axis) after the markings were drawn. The car position is measured on the middle crosssection. Locations are color coded. On most locations, drivers tend to slow down with the markings present. Triangle pointing down - inner lane in the curve; Trianlge pointing up-outer lane.

Acknowledgments: This work was supported by The Ministry of Education, Youth and Sports of the Czech Republic from the National Programme of Sustainability (NPU II); project IT4Innovations excellence in science - LQ1602. Also, this work was supported by TACR project "SMARTCarPark", TH03010529 and by the National Sustainability Programme I project of Transport R\&D Centre (LO1610), using the research infrastructure from the Operation Programme Research and Development for Innovations (ED2.1.00/03.0064).

\section{REFERENCES}

Babenko B., Yang M.-H., Belongie S. (2011), Robust object tracking with online multiple instance learning. IEEE Transactions on Pattern Analysis and Machine Intelligence, 33(8):1619-1632

Chrysler S., Re J., Knapp K., Funkhouser D., Kuhn B. (2009), Driver response to delineation treatments on horizontal curves on two-lane roads. Technical Report FHWA/TX-09/0-5772-1, Texas Transportation Institute

Dollár P., Appel R., Belongie S., Perona P., (2014) Fast feature pyramids for object detection. IEEE Transactions on Pattern Analysis and Machine Intelligence, 36(8):1532-1545

Fitzsimmons E., Souleyrette R., Nambisan S., (2013), Measuring horizontal curve vehicle trajectories and speed profiles:
Pneumatic road tube and video methods. Journal of Transportation Engineering - ASCE, 139(3):255-265

Glennon J. C., Weaver G. D. (2013), The relationship of vehicle paths to highway curve design. Technical Report 134-5, Texas Transportation Institute

Henriques J. F., Caseiro R., Martins P., Batista J., (2015), Highspeed tracking with kernelized correlation filters. IEEE Transactions on Pattern Analysis and Machine Intelligence.

Jamieson N. (2012), Clear zones, barriers and driving lines mitigating the effects of crashes on corners (horizontal curves). Technical Report 12-529B33, Opus International Consultants Ltd, Lower Hutt, New Zealand

Liu W., Anguelov D., Erhan D., Szegedy C., Reed S., Fu C.-Y., Berg A. C. (2016), SSD: Single shot multibox detector. In European Conference on Computer Vision, pages 21-37. Springer, 2016.

Murray S. (2017), Real-time multiple object tracking - A study on the importance of speed. CoRR, abs/1709.03572.

Pagano A., (1972), Appendix Q - Validation of intermediate criteria on rural horizontal curves. In National Cooperative Highway Research Program Report 130: Roadway Delineation Systems, pages 276-283, Washington, DC, National Research Council, Transportation Research Board.

Redmon J., Farhadi A., (2017), Yolo9000: Better, faster, stronger. In 2017 IEEE Conference Computer Vision and Pattern Recognition (CVPR), pages 6517-6525. IEEE.

Ren S., He K., Girshick R., Sun J., (2015), Faster R-CNN: Towards real-time object detection with region proposal networks. In Advances in neural information processing systems, pages 91-99.

Shi J. et al. (1994) Good features to track. In 1994 IEEE Computer Society Conference Computer Vision and Pattern Recognition, pages 593-600. IEEE.

Stimson I., McGee H., Kittelson A., Ruddy R. (2009), Field evaluation of selected delineation treatments on two-lane rural highways. Technical Report FHWA-RD-77-118, A.M. Voorhees \& Associates, Inc., McLean, Vir.

Viola P., Jones M. J., (2004), Robust real-time face detection. International journal of computer vision, 57(2):137-154

Zador P., Stein H., Wright P., Hall J., (1987), Effects of chevrons, postmounted delineators, and raised pavement markers on driver behavior at roadway curves. In Transportation Research Record 1114, pages 1-10. National Research Council, Transportation Research Board, Washington DC 


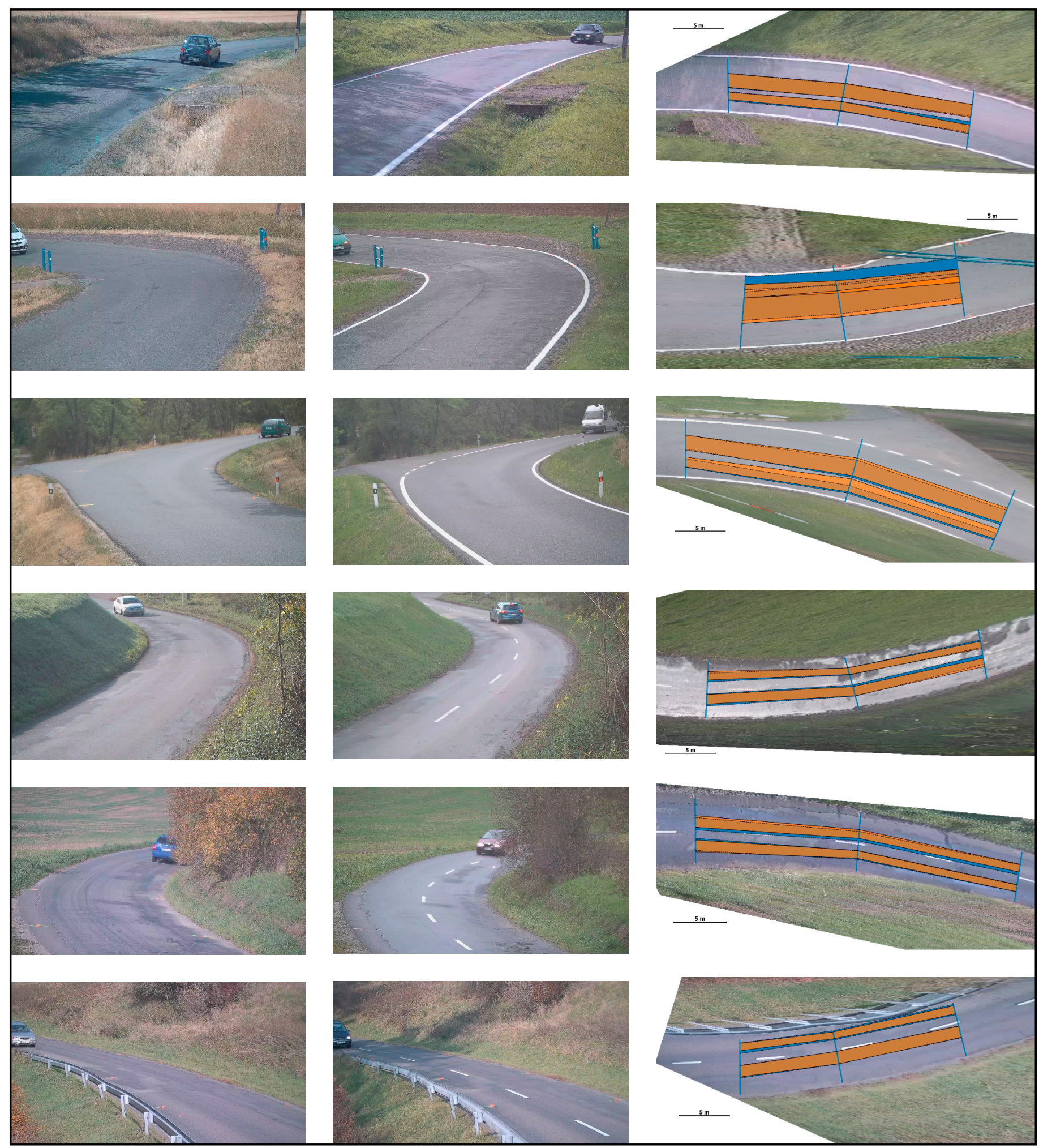

Fig. 4: Visualization of the measured localities. Left: camera view before lane makings applied, middle: view after lane markings applied, right: camera view projected on the ground plane computed from the calibration measurements (Sec. 3.1). Blue color marks the envelope of trajectories before road marking, orange: envelopes after road markings were drawn. 


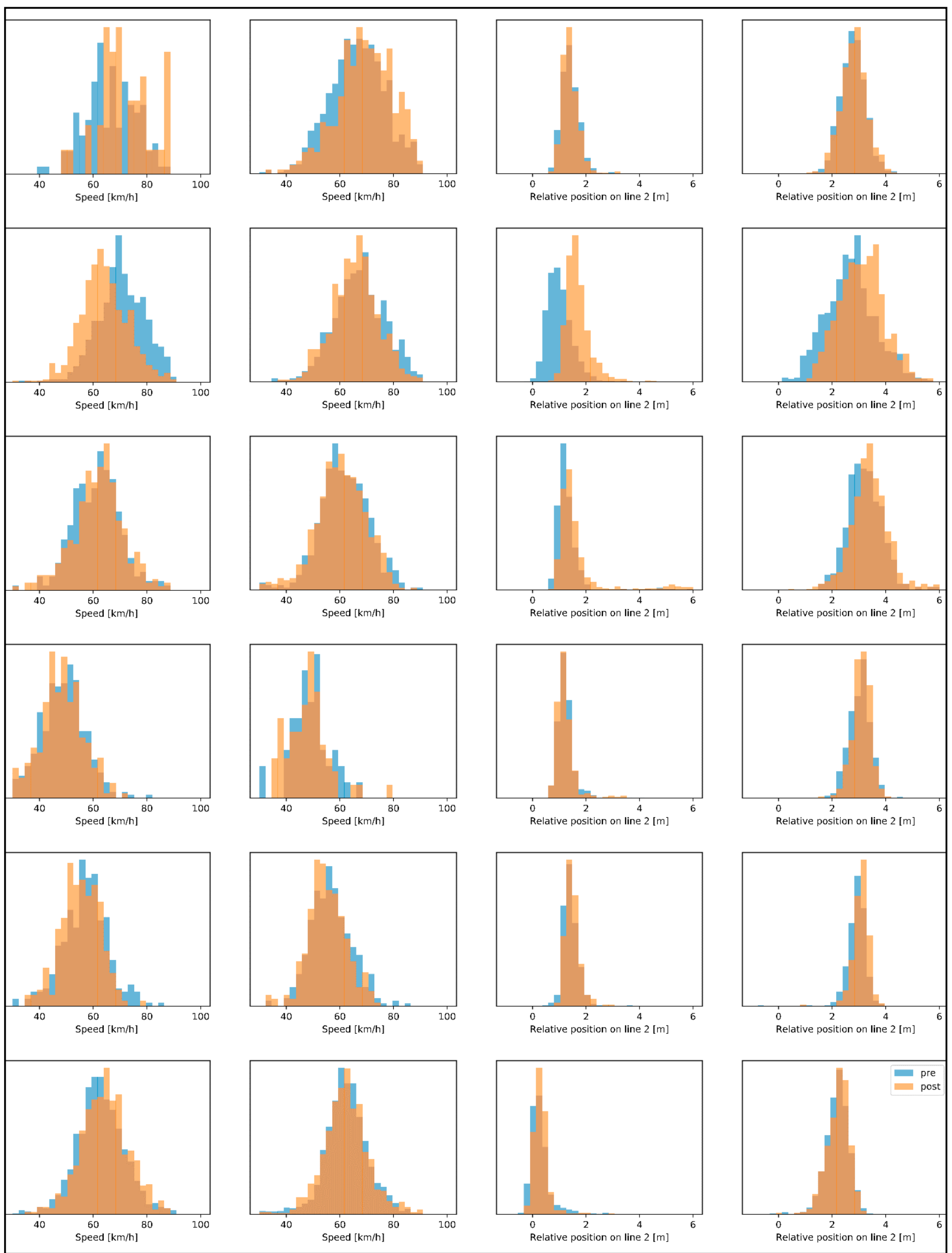

Fig. 5: Distribution changes of speeds (columns 1 and 2 for the inner and outer curve) and positions of cars (columns 3 and 4 for the inner and outer curve) for all locations (in rows). Blue: distribution before application of lines, orange: distribution after application of lines. 